inflammation can be therapeutically targeted by administration of synthetic methylated CpG-rich DNA oligonucleotides, ultimately associated with suppression of TLR9-mediated signaling responses and renal injury in experimental SLE/LN.

Conclusions: Collectively, our results implicate accumulation of unmethylated CpG-rich promoter DNA fragments in SLE-associated LN. Furthermore. these unmethylated $\mathrm{CpG}$-rich promoter DNA fragments causally contribute to TLR9mediated inflammation and renal fibrogenesis. Administration of methylated CpG-rich oligonucleotides antagonized intrarenal TLR9-mediated inflammatory signaling responses and fibrogenesis. Therefore, biomonitoring of CpG-rich promoter DNA fragments and modulation of intrarenal TLR9 signaling is a promising therapeutical target in SLE-associated LN.

Disclosure of Interest: None declared

DOI: 10.1136/annrheumdis-2017-eular.4493

\section{AB0155 LEARNING SLE PATHOLOGICAL MECHANISMS FROM MULTI OMICS PROFILES}

S.S. Pfister ${ }^{1}$, N. Kelley ${ }^{2}$, T. Schlitt ${ }^{3}$, A. Fernandez ${ }^{3}$, M. Sultan ${ }^{3}$, M. Hasan ${ }^{1}$, A. Mir ${ }^{1}$, J.S. Rush ${ }^{1} .{ }^{1}$ Autoimmunity, Transplantation and Inflammation; ${ }^{2}$ Analytical Sciences and Imaging; ${ }^{3}$ BioMarker Development, Novartis Institutes for BioMedical Research, Basel, Switzerland

Background: Precision medicine aims at providing intervention based on clinical and molecular stratification of patients, and is an important approach for targeting heterogeneous diseases. A diverse autoimmune disease is systemic lupus erythematosus (SLE), where dysregulation of several immune processes affects multiple organs. Fundamental for targeted treatment of such a heterogeneous disease is the identification of biomarkers predictive for the biological basis of clinical phenotypes.

Objectives: Despite recent progress, few markers for SLE are currently used in the clinic. In order to learn SLE pathological mechanisms and associated biomarkers, we obtained a diverse dataset from a cohort of active SLE patients (SLEDAI >6), including blood transcriptomics, serum proteomics, cytokines, and auto-antibody profiles. Integration of multi-omics data provides a rich dataset to explore associations between molecular and clinical readouts.

Methods: From a machine-learning perspective, biomarker discovery is defined as the process of selecting an optimal subset of variables for the prediction of parameters of interest. However, variable selection approaches are often underpowered for datasets that contain fewer samples than the number of variables. To overcome this problem we present a method based on L1 regularized regression and recursive variable elimination to generate networks of predictive markers across multiple data types.

Results: The proposed method allows us to graphically visualize the relationships among SLE phenotypes, and their molecular fingerprints. Identified networks of markers are validated by mapping to known biological pathways, and when available by comparison to independent patient cohorts. Despite the small number of patients $(n=20)$, we identify known pathological mechanisms, including a type I IFN gene signature, several cell type specific signatures, and potential novel markers of clinically defined SLE subtypes.

Conclusions: Systemic lupus erythematosus is a complex autoimmune disease characterized by a variety of clinical manifestations. While multi-omics profiles from SLE patients pose challenges because of their intrinsic high dimensionality, they also provide a unique insight into the molecular processes of disease. Our integrated analysis gives a novel perspective on the pathological mechanisms of clinical SLE phenotypes.

Disclosure of Interest: None declared

DOI: 10.1136/annrheumdis-2017-eular.5009

\section{AB0156 THYMIC STROMAL LYMPHOPOIETIN (TSLP) IN PRIMARY SJÖGREN'S SYNDROME AND RELATED LYMPHOMA: THE POSSIBLE CONTRIBUTION OF A NOVEL GROWTH FACTOR}

S. Gandolfo ${ }^{1,2}$, C. Fabro ${ }^{1,2}$, M. Bulfoni ${ }^{2,3}$, S. Russi ${ }^{4}$, P. Masolini ${ }^{1}$,

L. Quartuccio ${ }^{1,2}$, D. Cesselli ${ }^{2,3}, \mathrm{~S}$. De Vita ${ }^{1,2},{ }^{1}$ Rheumatology Clinic, Udine University Hospital S. Maria della Misericordia; ${ }^{2}$ Department of Medical and Biologic Sciences, University of Udine; ${ }^{3}$ Institute of Anatomic Pathology, Udine University Hospital S. Maria della Misericordia, Udine: ${ }^{4}$ Department of Biomedical Sciences and Human Oncology, University of Bari, Bari, Italy

Background: Primary Sjögren's syndrome (pSS) is an autoimmune lymphoproliferative systemic disease with a higher risk of non-Hodgkin B cell lymphoma (NHL) evolution. In SS, salivary gland (SG) epithelium plays a crucial role in initiating and perpetuating the autoimmune response. Thymic stromal lymphopoietin (TSLP) is both an epithelial and lymphopoietic cytokine involved in the maintenance of immune tolerance at interfaces between body and environment and in the regulation of lymphocytes homeostasis.

Objectives: To study TSLP in serum and SG biopsies of pSS patients stratified by the lymphoproliferative histopathologic status, from fully benign lesions (fbSS) to myoepithelial sialoadenitis (MESA) and to NHL, in order to evaluate a possible role of TSLP in SS pathogenesis and in lymphoma evolution.

Methods: Serum TSLP levels were determined by ELISA in pSS patients $(n=30$ : $12 \mathrm{fbSS}, 10 \mathrm{MESA}, 8 \mathrm{NHL}$ ) and in controls (healthy blood donors - HD $n=20$; non-autoimmune sicca without SS - nSS $n=10)$. TSLP was also studied by immunohistochemistry in SG biopsies of the same patients and nSS controls. Correlations with clinical and histopathologic parameters were performed. Of note, sequential samples were also included from three patients evolving from fbSS to NHL.

Results: TSLP serum levels were significantly higher in pSS compared to nSS $(p=0.03)$ and HD $(p=0.0002)$, with a progressive significant increase from fbSS to MESA ( $p=0.004$ ) and finally to NHL (NHL vs fbSS $p<0.0001$; NHL vs MESA $p=0.003$ ), where the increase was dramatic. This was observed also in metachronous samples from the three pSS patients evolving to NHL. A positive significant correlation between TSLP serum levels and disease activity assessed by ESSDAI was found $(p<0.0001)$.

Of note, in the affected tissue, TSLP showed an opposite pattern of expression than in the serum. Concerning the salivary epithelium, a declining expression of TSLP was shown from fbSS (expression was similar to nSS) to MESA and finally to NHL. Strikingly, however, the three pSS patients evolving to lymphoma were the only ones showing a low TSLP epithelial expression also at baseline. Concerning the glandular lymphoid infiltrate, the TSLP expression again decreased with progression to NHL. Again, the three pSS patients evolving to lymphoma were the only ones showing a low TSLP inflammatory expression also at baseline. Conclusions: Serum TSLP increases in pSS and correlates with lymphoma progression. Discrepancies are however observed between serum levels and tissue TSLP expression: while the baseline TSLP tissue expression is evident and similar to controls, tissue TSLP decreases with lymphoma progression. TSLP likely represents an additional growth factor and biomarker for pSS pathogenesis and lymphoproliferation.

Disclosure of Interest: None declared

DOI: 10.1136/annrheumdis-2017-eular.6450

\section{AB0157 TREX 1 MUTATION IN THE MEMBERS OF A FAMILY WITH SYSTEMIC LUPUS ERYTHEMATOSUS AND ANTIPHOSPHOLIPID SYNDROME}

S. Ugurlu ${ }^{1}$, I. Karacan ${ }^{2}$, H. Ozdogan ${ }^{1}$, A. Tolun ${ }^{3}$, E. Tahir Turanli ${ }^{4} .{ }^{1}$ Division of Rheumatology, Department of Internal Medicine, Cerrahpasa Medical Faculty, University of Istanbul; ${ }^{2}$ Graduate School of Science, Engineering and Technology, Molecular Biology, Biotechnology and Genetics Program, Dr. Orhan Ocalgiray Molecular Biology-Biotechnology and Genetics Research Centre, Istanbul Technical University; ${ }^{3}$ Department of Molecular Biology and Genetics, Boğaziçi University: ${ }^{4}$ Dr. Orhan Ocalgiray Molecular Biology Biotechnology and Genetics (MOBGAM) Research Centre, Istanbul Technical University, Istanbul, Turkey

Background: There are reports showing Three Prime Repair Exonuclease 1 (TREX1) mutations in atypical Systemic Lupus Erythematosus (SLE) patients. Objectives: Here we report a family with SLE and Antiphospholipid Syndrome (aPL) who are positive for TREX1 mutation and complicated with AA amyloidosis Methods: DNA samples were extracted from peripheral blood samples of two affected (mother and daughter) and two unaffected individuals of the same family. Exome sequencing was performed for the daughter and data was processed according to GATK Best Practices recommendations. Exome variants were used to search for a rare candidate variant causing the disease. Identified variant was screened in four family members using Sanger sequencing.

Results: The index case (mother) was a 63 year-old woman who had developed polyarthritis and recurrent cerebrovascular accident (CVA) at the age of 44. She was positive for ANA, anti-ds-DNA and IgG anti-cardiolipin antibodies. On anticoagulant therapy she still experiences frequent CVAs. The daughter who is 43 years old had experienced depression, non-erosive arthritis and alopecia at the age of 13. She was positive for ANA, dsDNA and ACA and was treated with hydroxychloroquine, prednisolone, and rituximab. She had attacks of deep vein thrombosis despite anticoagulant therapy. A renal biopsy was performed because of an increase in her creatinine level, with no proteinuria and normal urinary sediment, which revealed AA amyloidosis. She was heterozygous for $\mathrm{p}$. R202Q variant in MEFV gene. Further genetic testing was performed for the mother and the daughter as well as two other non-affected members of the family.

Candidate variant search in exome data resulted a novel c.2T $>A$ (p.M1?) variant in TREX1 gene. The variant was in heterozygous state for both affected members. Since the variant disrupts translation initiation codon (ATG/AAG), it is predicted to cause loss of complete protein production.

Conclusions: There is evidence that TREX1 is involved in the pathogenesis of SLE, especially with neuropsychiatric disease. Here we report a familial SLE and aPL syndrome complicated with AA amyloidosis, with a novel TREX1 variant. Such cases will increase our ability to understand the genetic spectrum of SLE and may allow the development of more effective therapies.

Disclosure of Interest: None declared

DOI: 10.1136/annrheumdis-2017-eular.6231 